\title{
Infall of planetesimals onto growing giant planets: onset of runaway gas accretion and metallicity of their gas envelopes
}

\author{
Masakazu Shiraishi and Shigeru Ida \\ Department of Earth and Planetary Sciences, Tokyo Institute of Technology, Ookayama, \\ Meguro-ku, Tokyo 152-8551, Japan \\ ida@geo.titech.ac.jp
}

\begin{abstract}
We have investigated the planetesimal accretion rate onto giant planets that are growing through gas accretion, using numerical simulations and analytical arguments. We derived the condition for gap opening in the planetesimal disk, which is determined by a competition between the expansion of the planet's Hill radius due to the planet growth and the damping of planetesimal eccentricity due to gas drag. We also derived the semi-analytical formula for the planetesimal accretion rate as a function of ratios of the rates of the Hill radius expansion, the damping, and planetesimal scattering by the planet. The predicted low planetesimal accretion rate due to gap opening in early gas accretion stages quantitatively shows that "phase 2," which is a long slow gas accretion phase before onset of runaway gas accretion, is not likely to occur. In late stages, rapid Hill radius expansion fills the gap, resulting in significant planetesimal accretion, which is as large as several $M_{\oplus}$ for Jupiter and Saturn. The efficient onset of runaway gas accretion and the late pollution may reconcile the ubiquity of extrasolar giant planets with metal-rich envelopes of Jupiter and Saturn inferred from interior structure models. These formulae will give deep insights into formation of extrasolar gas giants and the diversity in metallicity of transiting gas giants.
\end{abstract}

Subject headings: planetary systems: formation - solar system: formation

\section{Introduction}

Models of the interior structure of Jovian planets in our solar system suggest that Jupiter and Saturn would contain much more amount of heavy elements in their envelopes than that assuming the solar metallicity (Saumon \& Guillot 2004). This may imply that 
significant amount of planetesimals was accreted onto the planets together with gas accretion from the protoplanetary disk. However, the orbital calculations (e.g., Tanaka \& Ida 1997; Zhou \& Lin 2007) showed that the coupled effect of excitation of planetesimals' eccentricities due to gravitational scattering by the planet and their damping by aerodynamical and/or dynamical drag tends to open up a gap in the planetesimal disk, resulting in truncation of planetesimal infall onto the planet's gas envelope.

Pollack et al. (1996) a priori assumed the maximally efficient planetesimal accretion during gas accretion phase. As a result of increase in the planet's mass, the width of its feeding zone, which is proportional to cubic root of the mass, expands. They assumed that planetesimals in the expanded zones are accreted with the fastest rate for circular orbits of planetesimals. Their assumption can be consistent with the anticipated metal-rich envelopes of Jupiter and Saturn, but is inconsistent with the eccentricity excitation and gap formation shown by the above orbital integrations.

Furthermore, the assumption of the maximal planetesimal accretion results in long "phase 2" that is very inefficient gas accretion phase before onset of runaway gas accretion. As explained in $\S 2$, envelope contraction starts when core's mass $\left(M_{\mathrm{c}}\right)$ becomes larger than a critical core mass $\left(M_{\mathrm{c} \text {,hydro }}\right)$. For $M_{\mathrm{c}}>M_{\mathrm{c} \text {,hydro }}$, pressure gradient no more supports envelope gas hydrodynamically against the increased core's gravity (Mizuno 1980; Ikoma et al. 2000). Pollack et al. (1996) showed that heat generation due to the assumed planetesimal accretion associated with gas accretion supports the envelope quasi-hydrodynamically (in other words, it increases the critical core mass; eq. [1), after the onset of envelope contraction. The quasi-hydrodynamical state is called "phase 2" and it may last for more than Myrs. However, the inefficient gas accretion would be inconsistent with the ubiquity of extrasolar giant planets (Ida \& Lin 2008).

Recently, likelihood of phase 2 is re-addressed. Fortier et al. (2007) showed that even if the gap formation is neglected, more realistic planetesimal accretion rate based on oligarchic growth (Kokubo \& Ida 1998, 2002; Thommes et al. 2003) significantly suppresses the duration of phase 2. Zhou \& Lin (2007) showed that planetesimals around a protoplanet with $M_{\mathrm{c}} \sim M_{\text {c,hydro }}$ are gravitationally shepherded and cannot be accreted. It suggests nonexistence of phase 2. They also showed that the anticipated metal-rich envelopes of Jupiter and Saturn is not inconsistent with it, because such shepherding occurs only in early stages. The planet starts accreting planetesimals when its mass becomes comparable to that of gas giants because the planetesimals trapped in mean-motion resonances are released by resonance overlapping due to the planet's mass increase. The planetesimal accretion no more halts gas accretion onto such a massive planet. Through numerical simulations with two different simple gas accretion prescriptions, they estimated that total accreted mass can be 
as large as several earth masses.

The idea by Zhou \& Lin (2007) reconciled the efficient formation of gas giants with the anticipated metal-rich envelope. However, since they showed only numerical results with limited prescriptions for gas accretion, it is not clear that the accreted planetesimal mass for more realistic gas accretion rate is as much as that they obtained. Furthermore, they discussed suppression of phase 2 only qualitatively. As shown below, the planetesimal accretion rate does depend on gas accretion speed as well as the planet's mass. Hence, for evaluation of amount of planetesimal infall for more realistic gas accretion models and quantitative discussion on the suppression of "phase 2," general formulae for gas accretion rate as a function of the planet's mass $(M)$ and its increase rate $(\dot{M})$ is needed.

In the present paper, through orbital integrations, we clarify the physical mechanism to determine the planetesimal accretion rate and derive detailed semi-analytical formulae for the accretion rate as a function of $M$ and $\dot{M}$. We find that the total infall mass of planetesimals can be as much as several earth masses even for more realistic gas accretion models and quantitatively show that phase 2 is unlikely to occur.

The outline of this paper is as follows. We summarize gas accretion processes onto planets in $\S 2$. The method of our calculation and initial setup is described in $\S 3$. With artificial simple gas accretion models, we clarify intrinsic physics that determines the planetesimals accretion rate and derive semi-analytical formulae for the accretion rate ( $\$ 4.1$ to 4.3 ). Applying the formulae to realistic gas accretion models, we discuss the metallicity of Jupiter and Saturn envelope ( $\$ 4.4)$. We also discuss "phase 2 " and find that phase 2 is not likely to occur (§4.5). The conclusion is in $\S 5$.

\section{Gas Accretion Onto a Core}

As mentioned in $\S 1$, when the core mass becomes larger than a critical core mass, pressure gradient no more supports envelope gas hydrodynamically against the core's gravity and hydrostatic atmosphere does not exist. After that, heat generation due to gas envelope contraction itself supports the envelope against dynamical collapse, thus the envelope undergoes quasi-static contraction. The contraction allows gas inflow from the disk into Bondi radius of the planet, so that the contraction rate is almost equal to gas accretion rate of the planet.

Here, we briefly summarize the prescriptions for this process for later use. The critical core mass depends on planetesimal accretion rate onto the core $\left(\dot{M}_{\mathrm{c}}\right)$ and the grain opacity $\left(\kappa_{\mathrm{gr}}\right)$ associated with the disk gas. Based on a series of numerical models, Ikoma et al. (2000) 
found that the critical core mass for break-down of hydrostatic atmosphere is

$$
M_{\mathrm{c}, \text { hydro }} \simeq 10\left(\frac{\dot{M}_{\mathrm{c}}}{10^{-6} M_{\oplus} \mathrm{yr}^{-1}}\right)^{0.2-0.3}\left(\frac{\kappa_{\mathrm{gr}}}{\kappa_{\mathrm{gr}}^{\mathrm{P}}}\right)^{0.2-0.3} M_{\oplus},
$$

where $\kappa_{\mathrm{gr}}^{\mathrm{P}}\left(\sim 1 \mathrm{~cm}^{2} \mathrm{~g}^{-1}\right)$ is the grain opacity given by Pollack et al. (1985), who assumed dust grains with interstellar abundance and size distributions. Faster accretion and higher opacity (relatively large $\dot{M}_{\mathrm{c}}$ and $\kappa_{\mathrm{gr}}$ ) result in a warmer planetary atmosphere and an enhanced pressure gradient, so $M_{\text {c,hydro is larger (Stevenson 1982; Ikoma et al. 2000). }}$.

Pollack et al. (1996) assumed the most efficient planetesimal accretion induced by expansion of feeding zone due to increase of the planet mass, the rate of which is $\sim 10^{-6} M_{\oplus} \mathrm{yr}^{-1}$, for $M_{\mathrm{c}} \sim 10 M_{\oplus}$. When the planet with $M_{\mathrm{c}} \sim 10 M_{\oplus}$ becomes isolated consuming planetesimals in its feeding zone, gas envelope starts contraction and the induced planetesimal accretion from the expanded region of the feeding zone increases $M_{\mathrm{c} \text {,hydro }}$ up to $\sim M_{\mathrm{c}}$ (eq. [1]) and stall gas accretion. This self-regulated process works on more than Mrys until $M_{\mathrm{c}}$ exceeds $\sim 20 M_{\oplus}$. This is called "phase 2." However, as we show in $\S 4.5$, the rate of the planetesimal accretion induced by gas accretion is not generally large enough to maintain phase 2 . Then, gas accretion dominant phase starts.

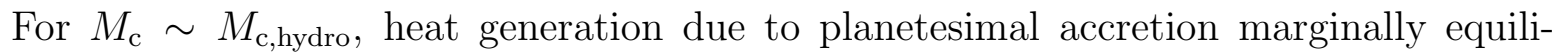
brates with the core's gravity. In the quasi-static contraction stage, heat generation due to envelope contraction marginally equilibrates with the gravity of the planet with total mass $M$ (including envelope mass). The Kelvin-Helmholtz contraction timescale is equivalent to planet mass increase timescale $\tau_{\mathrm{g}, \text { acc }}=M / \dot{M}$. Replacing $M_{\mathrm{c}}$ and $\dot{M}_{\mathrm{c}}$ by $M$ and $M / \tau_{\mathrm{g} \text {,acc }}$ in eq. (1), $\tau_{\mathrm{g}, \text { acc }}$ is given by

$$
\tau_{\mathrm{g}, \mathrm{acc}} \simeq 10^{7}\left(\frac{M}{10 M_{\oplus}}\right)^{-(2.3-4)}\left(\frac{\kappa_{\mathrm{gr}}}{\kappa_{\mathrm{gr}}^{\mathrm{P}}}\right) \text { yrs. }
$$

Detailed numerical simulations of quasi-static evolution of the gaseous envelope (Ikoma et al. 2000; Ikoma \& Genda 2006) show consistent results at the onset of runaway gas accretion in which the envelope and core masses are nearly equal. Although Podolak (2003) suggested $\kappa_{\text {gr }} \sim 0.01 \kappa_{\text {gr }}^{\mathrm{P}}$ through the numerical simulations of coagulation and sedimentation of dust grains in the atmosphere, the amount and size distribution of dust grains in the atmosphere are highly uncertain. Here, we adopt the results by Ikoma \& Genda (2006) with $\kappa_{\mathrm{gr}}=\kappa_{\mathrm{gr}}^{\mathrm{P}}$,

$$
\tau_{\mathrm{g}, \mathrm{acc}}=10^{6.5}\left(\frac{M}{10 M_{\oplus}}\right)^{-3.5} \mathrm{yrs},
$$

as a fiducial "realistic" gas accretion model. 
When eq. (3) is extrapolated to large $M\left(\gtrsim 100 \mathrm{M}_{\oplus}\right)$, it may give unrealistically fast supply of gas from the disk. Hence, we limit the gas accretion rate as bellow. Tanigawa \& Watanabe (2002) showed through two-dimensional local hydrodynamic simulations, the mass infall to the circumplanetary subdisk from the protoplanetary disk is limited by

$$
\frac{\dot{M}}{M} \simeq 6 \times 10^{-4} f_{g}\left(\frac{a}{5 \mathrm{AU}}\right)^{-1.5}\left(\frac{M}{M_{\oplus}}\right)^{0.3} \mathrm{yr}^{-1}
$$

where $f_{g}$ is a scaling factor for disk gas surface density defined by eq. (7). We use this limit with $f_{g}=0.7$.

Another limit is Bondi gas accretion, the rate of which is given by $\dot{M}=\pi r_{B}^{2} \rho_{g a s} c_{s}$, where $\rho_{\text {gas }}=\Sigma_{g} /(2 H)$ is the spatial density of gas disk and $H$ is the disk scale hight, $r_{B}=2 G M / c_{s}^{2}$ is the Bondi radius, $c_{s}=H \Omega$ is the sound speed and $\Omega=\sqrt{G M_{\odot} / a^{3}}$ is the Keplerian angular velocity. Adopting the temperature distribution in the limit of an optically thin disk (Hayashi 1981), $T=2.8 \times 10^{2}(r / 1 \mathrm{AU})^{-1 / 2} \mathrm{~K}$, the Bondi gas accretion limit is

$$
\frac{\dot{M}}{M}=0.7 \times 10^{-3}\left(\frac{a}{5 \mathrm{AU}}\right)^{-2}\left(\frac{M}{M_{\oplus}}\right) \mathrm{yr}^{-1} .
$$

As figure 1 shows, the timescale $(=M / \dot{M})$ in eq. (4) is generally longer than the Bondi accretion timescale, so an actual lower limit for gas accretion timescale is given by eq. (4).

\section{Calculation Setup}

\subsection{Orbital Integration}

We numerically calculate the orbital evolution of a swarm of planetesimals in the vicinity of a protoplanet's orbit embedded in a gaseous disk. The protoplanet grows accreting gas with a given rate. The planetesimals are treated as massless test particles and neglect their interactions. We assume that the protoplanet has a fixed circular orbit.

The planetesimals' orbits are affected by the gravitational force of the growing protoplanet and drag force from disk gas,

$$
\boldsymbol{f}_{\mathrm{gas}}=-\frac{\boldsymbol{v}-\boldsymbol{v}_{\mathrm{gas}}}{\tau_{\mathrm{damp}}}
$$

where $\boldsymbol{v}$ and $\boldsymbol{v}_{\text {gas }}$ are the velocity of a planetesimal and disk gas. The gas motion is a circular Keplerian motion. In some runs, we adopted slightly slower rotation speed of the disk gas due to radial pressure gradient in disk gas (e.g., Adachi et al. 1976), which induces 
inward migration of planetesimal orbits. However, we found that the inward migration hardly changed the results of planetesimal accretion rate onto the protoplanet. We here show the results without the inward migration. We set a damping timescale of the gas drag, $\tau_{\text {damp }}$ $(=e / \dot{e})$, as a constant parameter for all the planetesimals throughout a run in order to make clear the effect of the damping force.

We follow the prescription of gas surface density distribution by Ida \& Lin (2004),

$$
\Sigma_{g}=210 f_{g}\left(\frac{a}{5 \mathrm{AU}}\right)^{-3 / 2} \mathrm{~g} \mathrm{~cm}^{-2}
$$

where $f_{g}$ is the scaling parameter and $f_{g}=0.7$ corresponds to the gas surface density of the minimum mass solar nebular model, $\Sigma_{g, \text { MMSN }}$ (Havashi 1981). For simplicity, we neglect a gap in the gas disk, which may be opened up by the perturbations from a massive protoplanet (e.g., Lin \& Papaloizou 1993) and assume the above unperturbed $\Sigma_{g}$ everywhere. With this $\Sigma_{g}$, a given value of $\tau_{\text {damp }}$ corresponds to individual planetesimal mass (Adachi et al. 1976; Tanaka \& Ida 1999),

$$
m=3 \times 10^{17} f_{g}^{3}\left(\frac{e}{0.1}\right)^{3}\left(\frac{\tau_{\mathrm{damp}}}{10^{5} \mathrm{yr}}\right)^{3}\left(\frac{\rho_{\mathrm{pl}}}{1 \mathrm{~g} \mathrm{~cm}^{-3}}\right)^{-2}\left(\frac{a}{5 \mathrm{AU}}\right)^{-39 / 4} \mathrm{~g},
$$

where $a, e$, and $\rho_{\mathrm{pl}}$ are semi-major axis, eccentricity, and material density of planetesimals, respectively. If gravitational drag (e.g., Tanaka \& Ward 2004) is considered in stead of aerodynamical gas drag,

$$
m=4.5 \times 10^{26} f_{g}^{-1}\left(\frac{\tau_{\mathrm{damp}}}{10^{5} \mathrm{yr}}\right)^{-1}\left(\frac{a}{5 \mathrm{AU}}\right)^{2} \mathrm{~g},
$$

although in this case, interactions among the planetesimals could be important.

The orbits of planetesimals are numerically integrated by using the fourth-order Hermite scheme (Makino \& Aarseth 1992) with the hierarchical timestep (Makino 1991). The equation of motion of particle $k$ is given by

$$
\frac{d^{2} \boldsymbol{r}_{k}}{d t^{2}}=-G M_{\odot} \frac{\boldsymbol{r}_{k}}{\left|\boldsymbol{r}_{k}\right|^{3}}-\frac{G M\left(\boldsymbol{r}_{k}-\boldsymbol{r}\right)}{\left|\boldsymbol{r}_{k}-\boldsymbol{r}\right|^{3}}-\frac{G M \boldsymbol{r}}{|\boldsymbol{r}|^{3}}+\boldsymbol{f}_{\text {gas }}
$$

where $M$ and $\boldsymbol{r}$ is the mass and position of the protoplanet. The first term to the last one represent the gravity from the central star, the gravitational perturbation from the protoplanet, the indirect term and the gas drag force, respectively. We set $M_{*}=M_{\odot}$.

When a planetesimal contacts the surface of the protoplanet, the planetesimal is removed after recording the collision. The planet mass is unchanged. The physical radius of a 
protoplanet is determined by its mass and internal density $\rho$ as

$$
R=\left(\frac{3 M}{4 \pi \rho}\right)^{1 / 3}
$$

We set $\rho=1 \mathrm{gcm}^{-3}$ in all simulations. The dependence of the planetesimal accretion rates on $\rho$ will be discussed in $\S 4.4$.

Although we neglect gravitational forces of planetesimals, mass of planetesimals is specified in order to calculate the amount of mass accretion onto the protoplanet (regarding "effective" mass for gas drag force, see below). Assuming equal-mass planetesimals, they are initially distributed in the range $a_{\text {in }}<a<a_{\text {out }}$ to satisfy the surface mass density

$$
\Sigma_{d}=3.8 f_{d}\left(\frac{a}{5 \mathrm{AU}}\right)^{-3 / 2} \mathrm{~g} \mathrm{~cm}^{-2},
$$

where $f_{d}$ is a scaling factor. As is the case for $f_{g}, f_{d}=0.7$ corresponds to MMSN. The inner and outer boundaries are $a_{\text {in }}=a_{p}\left(1-5 h_{\mathrm{f}}\right)$ and $a_{\text {out }}=a_{p}\left(1+10 h_{\mathrm{f}}\right)$, where $a_{p}$ is the semi-major axis of the protoplanet and $h_{\mathrm{f}}$ is the reduced Hill radius for final mass of the planet $\left(M_{\mathrm{f}}\right)$. The reduced Hill radius of a protoplanet $h$ is Hill radius $r_{\mathrm{H}}$ scaled by $a_{p}$,

$$
h=r_{\mathrm{H}} / a_{p}=\left(\frac{M}{3 M_{*}}\right)^{1 / 3} .
$$

In all numerical simulations, we adopt $a=5 \mathrm{AU}, M_{\mathrm{f}}=M_{J}$ (Jupiter mass), and $f_{d}=$ $f_{g}$. Accordingly, $h_{\mathrm{f}}=6.8 \times 10^{-2}, a_{\text {in }}=3.3 \mathrm{AU}$, and $a_{\text {out }}=8.4 \mathrm{AU}$. We will derive the dependences on $a, M_{\mathrm{f}}$, and $f_{d}\left(=f_{g}\right)$ by analytical arguments and discuss the results with different parameter values. Total mass of planetesimals within the region $a_{\text {in }}<a<a_{\text {out }}$ is $\sim 20 f_{d} M_{\oplus}$. The number of planetesimals in most runs is $N=20000$. With an assumption that planetesimals have an equal mass, their individual mass is $m_{p l} \simeq 1.1 \times 10^{-3} f_{d} M_{\oplus}=$ $6.6 \times 10^{24} f_{d}$ g. In our simulations, we specify $\tau_{\text {damp }}=10^{6}, 10^{5}, 10^{4}$ yrs and $\infty$ (gas-free case), independent of the values of $m_{p l}$. Except for the gas-free case, the above values of $m_{p l}$ is much larger than the values in eq. (8) for the given $\tau_{\text {damp }}$, so planetesimals that we use correspond to "super particles" representing many smaller planetesimals. Since we neglect interactions among planetesimals, such "super particles" treatment is not inconsistent. Initial eccentricity and inclination of planetesimals are taken as $e_{0}=i_{0}=0.001$ for all simulations. Since $e$ and $i$ are quickly pumped up by perturbations from the protoplanet, the choice of $e_{0}$ and $i_{0}$ does not affect results. 


\subsection{Growth of a Protoplanet}

Since we consider the phase after isolation of protoplanets, we assume that the growth of the protoplanets is dominated by accretion of surrounding disk gas but not by planetesimals. As we will show later, this assumption is valid, because amount of accreted gas is much larger than the anticipated amount of accreted planetesimals.

In $\S 2$, we described the prescription for gas accretion. In the numerical simulations, we use simple artificial gas accretion models in order to make clear what conditions regulate the planetesimal accretion rate. From the results with the artificial models, we derive semi-analytical formulae for the planetesimal accretion in general forms (§4.3). Applying the formulae to the more realistic gas accretion rate in $\S 2$, we calculate the total mass of planetesimal infall into the envelope of Jupiter and Saturn in $§ 4.4$.

The simple artificial gas accretion models are expressed by

$$
\frac{d M}{d t} \equiv \alpha M^{p}
$$

where $\alpha$ is the integration constant determined by the boundary condition. We set the condition as $M=M_{0}$ for $t=0$ and $M=M_{\mathrm{f}}$ for $t=t_{\mathrm{f}}$. Following Zhou \& Lin (2007), we set the protoplanet at $5 \mathrm{AU}$ with its initial mass $M_{0}=5.67 M_{\oplus}$ and final mass $M_{\mathrm{f}}=M_{J}$ (Jupiter

mass). We adopt $t_{\mathrm{f}}=10^{5} \mathrm{yr}$ for numerical simulation, following their nominal cases. The growth with $p=2$ and 0 correspond to the Bondi and linear models in Zhou \& Lin (2007). Figure 2 shows the evolution of the mass of a protoplanet by accreting gas for $p=2,1,0,-2$. Here we assume that $M_{0}>M_{\mathrm{c} \text {,hydro }}$. The consistency of the assumption is checked in $\S 4.5$.

\section{Results of Orbital Calculation}

\subsection{Overall Evolution}

Figure 3 shows the snapshots of the distributions of planetesimals on the $b$-e/h plane, where $h$ is defined by eq. (13). The scaled orbital separation $b$ is defined by

$$
b=\frac{a-a_{p}}{h a_{p}}
$$

where $a_{p}$ is semimajor axis of the protoplanet. The protoplanet is fixed at the origin (i.e., $b=0$ and $e=0)$. The growth rate of the protoplanet $\dot{M}$ is $\propto M^{2}$. To avoid busy plots, we show only 1000 planetesimals in this figure. We integrate the evolution of planetesimals for $3 \times 10^{5} \mathrm{yrs}$. Since $t_{\mathrm{f}}=10^{5} \mathrm{yrs}$, we set $\dot{M}=0$ for $t=1-3 \times 10^{5} \mathrm{yrs}$, which corresponds to 
termination of gas accretion due to gap formation in the gas disk, although we neglect the effect of gas density depletion on drag force. The damping timescale is $\tau_{\text {damp }}=10^{4} \mathrm{yr}$.

In figure 3, we also drew the Jacobi energy $E_{J}$,

$$
E_{J}=\frac{1}{2}\left((e / h)^{2}+(i / h)^{2}\right)-\frac{3}{8} b^{2}+\frac{9}{2}+O(h) .
$$

In the figure, we also include higher order terms of $h$ in $E_{J}$. In the circular restricted threebody problem, $E_{J}$ is conserved between before and after scattering by the protoplanet (on average, both $\left(e^{2}+i^{2}\right)$ and $b^{2}$ increase). Since only planetesimals with $E_{J} \geq 0$ can enter the Hill sphere of the protoplanet (e.g., Havashi et al. 1977), we regard the region $E_{J}>0$ as the feeding zone of the protoplanet. When $e / h, i / h \lesssim 1$, the width of feeding zone is $b \simeq 2 \sqrt{3}$.

In the top panel $(t=1000 \mathrm{yrs})$, the planetesimals in the vicinity of the protoplanet are scattered and their $e$ and $b$ increase along a constant $E_{J}$ curve. The planetesimal eccentricities $e$ are damped in the panel of $t=3 \times 10^{4} \mathrm{yrs}$ because of $\tau_{\text {damp }}=10^{4} \mathrm{yrs}$. Since the gas drag damps $e$ keeping $b$ almost constant, all the planetesimals except for those trapped in horseshoe orbits go out of the feeding zone. As the protoplanet grows up, its feeding zone expands. Since $b \propto M^{-1 / 3}, b$ of planetesimals decreases with time, but scattering opposes it. Since $\dot{M} \propto M^{2}$, the expansion accelerates with time. Eventually, the expansion overwhelms the gap opening due to a coupling effect of scattering and gas drag damping, so that planetesimals go into the feeding zone in the panel of $t=1 \times 10^{5} \mathrm{yrs}$. After $t=1 \times 10^{5} \mathrm{yrs}$, the increase of $M$ stopped, so a gap in the planetesimal disk is again produced (the bottom panel). Planetesimals that have sufficiently large $b$ are captured in proper mean motion resonances. Although we neglect inward migration due to slightly slower rotation of gas than Keplerian rotation due to pressure gradient, damping of $e$ results in small decrease in $b$ due to angular momentum conservation. Such inward migration causes resonance trapping. The evolution of the gap width is consistent with the result by Zhou \& Lin (2007).

Thus, after initial relaxation, planetesimals are shepherded and planetesimal accretion rate is very low, until efficient planetesimal accretion re-starts in late stage. Zhou \& Lin (2007) showed through orbital simulation of planetesimals that planetesimal accretion occurs only in late stage of gas accretion. They suggested that in late stage, planet's mass becomes large and the mean motion resonances overlap to release planetesimals captured in the resonances. So, they concluded that mass of the protoplanet controls the accretion rate of planetesimals onto the protoplanet. This effect indeed determines supply of planetesimals to the regions near the feeding zone. However, whether the planetesimals near the feeding zone are shepherded or not is determined by gap opening that is a result of competing processes of the feeding zone expansion and scattering/damping, so the values of $\dot{M}$ play an important role as well as $M$. 


\subsection{Dependence of Planetesimal Accretion Rate on Planet's Mass}

The evolution of the planetesimal accretion rate for $\tau_{\text {damp }}=10^{6}, 10^{5}, 10^{4}$ yrs and gasfree case is plotted as a function of $M$ in Figure 4. The four lines in each panel represent various gas accretion models $(p=2,1,0,-2)$. Initial mass of the protoplanet $M_{0}$ is set as $5.67 \mathrm{M}_{\oplus}$. Starting with 20,000 planetesimals (i.e., planetesimal mass $\sim 1.1 \times 10^{-3} f_{d} M_{\oplus}$ ), we calculated for $10^{5} \mathrm{yrs}$ (the growth timescale $t_{\mathrm{f}}=10^{5} \mathrm{yrs}$ ).

To see the dependence on $M$ more clearly, we plot the scaled planetesimal accretion rate $\dot{M} / R^{2}$, where $R$ is the physical radius of the protoplanet. Through the numerical simulations, we found that planetesimals are likely to experience 2-D accretion rather than 3 -D. The 2-dimensional accretion rate is

$$
\frac{d M}{d t} \sim 2 R \Sigma_{d}\left(\frac{v_{\mathrm{esc}}}{v_{\mathrm{rel}}}\right) v_{\mathrm{rel}}=\sqrt{\frac{32 \pi \mathrm{G} \rho}{3}} \Sigma_{d} R^{2},
$$

where $\Sigma_{d}, v_{\text {esc }}=\sqrt{2 \mathrm{GM} / R}$ and $v_{\text {rel }}$ are the surface density of the planetesimals, escape velocity from the protoplanet's surface and relative velocity between the protoplanet and planetesimals, respectively. The scaled accretion rate $\left(\dot{M} / R^{2}\right)$ is determined by effective $\Sigma_{d}$ in the feeding zone for fixed internal density of the protoplanet $(\rho)$. In our simulations, the total mass of the planetesimals is not significantly decreased, so the effective $\Sigma_{d}$ is determined by scattering by the planet, gas drag, and Hill radius expansion due to the planet growth.

Figure 4 shows that the planetesimal accretion rates for $f_{d}=0.7$. The planetesimal accretion rates as a function of $M$ depends on the parameter $p$. For $p=-2$ and 0 , the scaled planetesimal accretion rate decreases with $M$, which suggests that a gap in the planetesimal disk is formed when $M$ becomes large. On the other hand, for $p=2$, the protoplanet may grow so fast in the late stage that the feeding zone expansion overwhelms the gap formation, as shown in figure 3. The dependence on $p$ implies that the accretion rate is not a function solely of $M$, but depends on $\dot{M}$ as well as $M$, because $p$ determines the $\dot{M}-M$ relation.

\subsection{Dependence of Planetesimal Accretion Rate on Gap Formation Parameters}

Here we show that competition among the feeding zone expansion, scattering, and eccentricity damping regulates flux of planetesimals across the boundaries of the feeding zone $\left(E_{J}=0\right)$, that is, the planetesimal accretion rate. We consider change rates of $b^{2}$ and $(e / h)^{2}$ of planetesimals (we neglect the contribution from $i$ because $i$ is usually correlated to $e$ and $i<e)$. 
Evolution of planetesimals on the $b^{2}-(e / h)^{2}$ space due to gravitational scattering by the protoplanet, damping of eccentricity by gas drag, and expansion of Hill radius by mass increase of the protoplanet is expressed by the change rates, $v_{\text {scat }}, v_{\text {damp }}$, and $v_{\mathrm{H}}$, on the plane. Since $b \propto h^{-1} \propto M^{-1 / 3}$,

$$
v_{\mathrm{H}} \equiv \frac{d b^{2}}{d t}(\text { growth })=(b h)^{2} \frac{d h^{-2}}{d t}=-\frac{2}{3} b^{2} \frac{\dot{M}}{M} \simeq-\frac{8}{\tau_{\mathrm{g}, \mathrm{acc}}},
$$

where $\tau_{\mathrm{g}, \text { acc }}=M / \dot{M}$ is the timescale of planet mass increase. In the last equation, we used $b \simeq 2 \sqrt{3}$, which is the location of the feeding zone for $e / h \lesssim 1$, for simplicity. With $\tau_{\text {damp }}=e / \dot{e}$,

$$
v_{\text {damp }} \equiv \frac{1}{2} \frac{d(e / h)^{2}}{d t}(\text { damping })=-\frac{(e / h)^{2}}{\tau_{\text {damp }}} .
$$

The factor $(1 / 2)$ in the definition is added for the more simple form of the final expression and better fit with numerical results. Evolution due to the scattering is increase of $b^{2}$ and $(e / h)^{2}$ on average, along a constant $E_{J}$ curve $\left(E_{J} \sim 0\right)$. The corresponding change rate is

$$
v_{\text {scat }} \equiv \frac{d b^{2}}{d t}(\text { scattering })=\frac{4}{3} \frac{d(e / h)^{2}}{d t}(\text { scattering }) .
$$

Assuming long-range gravitational interaction with $(e / h) \lesssim 1$, linear calculation Goldreich \& Tremaine 1982; Hasegawa \& Nakazawa 1990) showed that $b$ of a planetesimal is increases by $\delta b \simeq 30 b^{-5}$ during each encounter. Numerical calculation showed that for $b \sim 3-4, \delta b$ is overestimated by a factor $\sim 10($ Ida 1990). Since the scattering occurs at every synodic time $T_{\mathrm{syn}} \simeq 2 \pi a_{p} /\left(\frac{3}{2} b r_{\mathrm{H}} \Omega_{K}\right)$

$$
v_{\text {scat }}=2 b \frac{d b}{d t}(\text { scattering }) \simeq 2 b \frac{0.1 \delta b}{T_{\mathrm{syn}}} \simeq \frac{6}{b^{4}} \frac{(3 / 2) b h}{T_{\mathrm{K}}} \simeq 0.22 \frac{h}{T_{\mathrm{K}}},
$$

where $T_{\mathrm{K}}=2 \pi / \Omega_{\mathrm{K}}$ is Keplerian period and $b \simeq 2 \sqrt{3}$ is again used.

Since the feeding zone is determined by the values of $E_{J}$ and the scattering does not change the values, the condition of gap opening would be $v_{\text {damp }} \gtrsim v_{\mathrm{H}}$. If inward migrations of planetesimals due to gas drag or type I migration of the protoplanet is considered but growth of a protoplanet is neglected, the gap formation condition is similarly derived, replacing $v_{\mathrm{H}}$ by $d b^{2} / d t$ due to gas drag (Tanaka \& Ida 1997) or type I migration (Tanaka \& Ida 1999). These effects can suppress growth of the protoplanets before they attain their isolation masses (Tanaka \& Ida 1997, 1999).

When $v_{\text {damp }} \lesssim v_{\mathrm{H}}$, the gap is not created and planetesimals are engulfed by the expanding feeding zone. The engulfment rate would be determined by $v_{\mathrm{H}} / v_{\text {scat }}$, because $v_{\mathrm{H}}$ and 
$/ v_{\text {scat }}$ have opposite directions to each other in the $b^{2}$ components. Thus, it is expected that for $v_{\text {damp }}<v_{\mathrm{H}}$, the accretion rate would be regulated by

$$
\xi \equiv\left|\frac{v_{\mathrm{H}}}{v_{\mathrm{scat}}}\right| \simeq 37 h^{-1} \frac{T_{\mathrm{K}}}{\tau_{\mathrm{g}, \mathrm{acc}}} \simeq 4.1\left(\frac{a_{p}}{5 \mathrm{AU}}\right)^{3 / 2}\left(\frac{M}{M_{\oplus}}\right)^{-1 / 3}\left(\frac{\tau_{\mathrm{g}, \mathrm{acc}}}{10^{4} \mathrm{yrs}}\right)^{-1},
$$

while for $v_{\text {damp }}>v_{\mathrm{H}}$, the accretion rate would be regulated by

$$
\eta \equiv\left|\frac{v_{\mathrm{H}}}{v_{\mathrm{damp}}}\right| \simeq \frac{8}{(e / h)^{2}} \frac{\tau_{\mathrm{damp}}}{\tau_{\mathrm{g}, \mathrm{acc}}} \simeq 0.8\left(\frac{\tau_{\mathrm{damp}}}{10^{4} \mathrm{yrs}}\right)^{1 / 2}\left(\frac{\tau_{\mathrm{g}, \mathrm{acc}}}{10^{4} \mathrm{yrs}}\right)^{-1}\left(\frac{M}{M_{\oplus}}\right)^{-1 / 6}\left(\frac{a_{p}}{5 \mathrm{AU}}\right)^{3 / 4}
$$

where we used eq. (39) in Appendix for $(e / h)^{2}$. Since $\tau_{\mathrm{g} \text {,acc }}=M / \dot{M}$ and $\tau_{\text {damp }}$ and $h$ are functions of $M$, the planetesimal accretion rate would depend on $\dot{M}$ as well as $M$. We show that the numerical results agree with the above argument and derive formulae for the planetesimal accretion rate as a function of $\xi$ and $\eta$.

Figure 5 shows the evolution of the scaled planetesimal accretion rate as a function of $\xi=v_{\mathrm{H}} / v_{\text {scat }}$ for $p=2,1,0$ and -2 in the cases of $\tau_{\text {damp }}=10^{6}, 10^{5}, 10^{4}$ yrs and gas-free case. As suggested in the above discussion, figure 5 shows that in the ranges of $\eta>1$ [equivalently, $\left.\xi>5\left(\tau_{\text {damp }} / 10^{5} \mathrm{yr}\right)^{-1 / 2}\left(M / M_{\oplus}\right)^{-1 / 6}\left(a_{p} / 5 \mathrm{AU}\right)^{-3 / 4}\right]$, the scaled planetesimal accretion rate is independent of planet gas accretion models with different $p$ (different $\dot{M}-M$ relations) and different $\tau_{\text {damp. }}$. This confirms that the accretion rate is determined by $\xi$ for $\eta>1$ (non-gap cases). The planetesimal accretion rate in this case is given by

$$
\frac{d M_{\text {solid }}}{d t}=10^{\beta}\left(\frac{R}{R_{\oplus}}\right)^{2} f_{d}\left(\frac{v_{\mathrm{H}}}{v_{\text {scat }}}\right)^{\alpha} \mathrm{M}_{\oplus} \mathrm{yr}^{-1}
$$

with $\alpha \simeq 0.8$ and $\beta \simeq-6$ that are obtained from our numerical results by the least square fitting. The fitting line, eq. (24), is expressed by thick solid lines in the plots. For $\eta<1$, the accretion rate declines, which corresponds to gap opening in the planetesimal disk.

Figure [6] shows the evolution of the scaled planetesimal accretion rate as a function of $\eta=v_{\mathrm{H}} / v_{\text {damp }}$. In the range of $\eta<1$, the scaled planetesimal accretion rate is independent of planet accretion models with different $\dot{M}-M$ relations and different $\tau_{\text {damp. This confirms that }}$ the accretion rate is determined by $\eta$ for $\eta<1$. From our numerical results, the planetesimal accretion rate in this case is given by

$$
\frac{d M_{\text {solid }}}{d t}=10^{\beta}\left(\frac{R}{R_{\oplus}}\right)^{2} f_{d}\left(\frac{v_{\mathrm{H}}}{v_{\mathrm{damp}}}\right)^{\alpha} \mathrm{M}_{\oplus} \mathrm{yr}^{-1}
$$

with $\alpha \simeq 1.4$ and $\beta \simeq-6$. 
When the planet's mass has grown to $M$, the total mass of planetesimals that infall in the envelope $\left(M_{\text {solid }}(M)\right)$ is obtained by integrating

$$
\frac{d M_{\text {solid }}}{d M}=\frac{d M_{\text {solid }}}{d t} \frac{\tau_{\mathrm{g}, \text { acc }}}{M}
$$

from 0 to $M$. In figure 7, $M_{\text {solid }}(M)$ evaluated by the above semi-analytical formulae is compared with that obtained by orbital calculations for individual gas accretion models in cases of $\tau_{\text {damp }}=10^{6} \mathrm{yrs}, 10^{5} \mathrm{yrs}$ and $10^{4} \mathrm{yrs}$. The semi-analytical formulae well reproduce the results of orbital calculations except for early stages in which $M_{\text {solid }}$ is so small that statistical fluctuation is large. The formulae also reproduce numerical results in their figure $9 \mathrm{a}$ in Zhou \& Lin (2007).

\subsection{Application to Jupiter and Saturn}

In the preceding subsection, we investigated planetesimal accretion onto growing protoplanets with artificial gas accretion models and obtained semi-empirical formulae of the planetesimal accretion rate. Applying this formulae to the more realistic gas accretion models in $\S 2$, we discuss the metallicity of envelopes of Jupiter and Saturn.

Integrating eq. (26) with eq. (3) to $M_{\mathrm{f}}$, we estimate total mass of the accreted planetesimals in the cases of Jupiter $\left(M_{\mathrm{f}}=318 M_{\oplus}, a_{p}=5.2 \mathrm{AU}\right)$ and Saturn $\left(M_{\mathrm{f}}=95 M_{\oplus}\right.$, $a=9.55 \mathrm{AU})$. The evolution of $M_{\text {solid }}$ is plotted in figure 8 . The three curves show the results with $\tau_{\text {damp }}=10^{4}, 10^{5}$ and $10^{6}$ yrs. It is likely that gas giants were inflated during gas accretion phase. For a fixed $M, d M / d t \propto f_{d} \sqrt{\rho} R^{2} \propto f_{d} \sqrt{R}$ (eq. [17]). In the figure, we plot the accreted planetesimal mass $M_{\text {solid }}^{*}$ for $R=2 R_{1}$ and $f_{d}=2$, where $R_{1}$ is the physical radius for mass $M$ and $\rho=1 \mathrm{gcm}^{-3}$. For other $R$ and $f_{d}$, the accreted mass is $M_{\text {solid }}=\left(R / 2 R_{1}\right)^{1 / 2}\left(f_{d} / 2\right) M_{\text {solid }}^{*}$.

All the results show similar qualitative features of evolution of $M_{\text {solid }}$. Planetesimal accretion is inhibited in early stages by gap formation, but rapid planetary growth due to gas accretion in later stages allows planetesimal accretion. With $\tau_{\text {damp }}=10^{6} \mathrm{yrs}, M_{\text {solid }} \simeq$ $6\left(R / 2 R_{1}\right)^{1 / 2}\left(f_{d} / 2\right) M_{\oplus}$ both for Jupiter and Saturn. For shorter $\tau_{\text {damp }}, M_{\text {solid }}$ is smaller due to easier gap formation. We also did calculations starting from different core masses. The resultant $M_{\text {solid }}$ hardly changed, because $d M_{\text {solid }} / d M$ is negligibly small when $M$ is small and gap is opened. The amount of predicted $M_{\text {solid }}$ can be as large as that inferred from the internal structure model Saumon \& Guillot (2004), if the planets are inflated and/or relatively large $f_{d}$ is considered.

For the same $M, \rho$ and $f_{d}, M_{\text {solid }}$ is larger for larger $a_{p}$. Although Saturnian mass is 
$1 / 3$ of Jovian mass, our model predicts that the mass of planetesimals falling into Saturnian envelope is comparable to that into Jovian envelope. More detailed internal structure models will test our prediction.

\subsection{Phase 2}

So far, we have assumed that gas accretion immediately starts when $M_{\mathrm{c}}$ exceeds $M_{\mathrm{c} \text {,hydro }}$ without undergoing "phase 2." In the previous subsection, we predicted the planetesimal accretion rate as a function of planetary mass based on the realistic gas accretion model. With this accretion rate, we show that "phase 2" is not likely to occur.

In the nominal model (J1 model) in Pollack et al. (1996) $, f_{d} \simeq 2.5, a_{p}=5.2 \mathrm{AU}$ and $M_{\mathrm{c}} \simeq 10 M_{\oplus}$. Then, they found that $\dot{M}_{\mathrm{c}} \simeq 10^{-6} M_{\oplus} / \mathrm{yr}$ is maintained during "phase 2 " with their maximally efficient planetesimal accretion model. As shown in eq. (11), this $\dot{M}_{\mathrm{c}}$ can marginally support gas envelope around a $10 M_{\oplus}$ core.

First, we derive the condition for gap opening with a realistic $\tau_{\text {g,acc }}$ given by eq. (3). Substituting eq. (3) into eq. (23),

$$
\eta \simeq 0.8 \times 10^{-6}\left(\frac{\tau_{\text {damp }}}{10^{4} \mathrm{yrs}}\right)^{1 / 2}\left(\frac{M}{M_{\oplus}}\right)^{3.3}\left(\frac{a_{p}}{5 \mathrm{AU}}\right)^{3 / 4}
$$

With $a_{p}=5.2 \mathrm{AU}, M \sim M_{\mathrm{c}} \sim 10 M_{\oplus}$ and $\tau_{\text {damp }}=10^{6}$ yrs, we obtain $\eta \simeq 2 \times 10^{-2} \ll 1$. Then, the gap should be opened up. Our formula for $\eta<1$ gives

$\dot{M}_{\text {solid }} \simeq 2.2 \times 10^{-6} f_{d}\left(\frac{\rho_{\mathrm{p}}}{1 \mathrm{gcm}^{-3}}\right)^{-1 / 6}\left(\frac{\tau_{\mathrm{damp}}}{10^{4} \mathrm{yrs}}\right)^{7 / 10}\left(\frac{\tau_{\mathrm{g}, \mathrm{acc}}}{10^{4} \mathrm{yrs}}\right)^{-7 / 5}\left(\frac{M}{M_{\oplus}}\right)^{13 / 30}\left(\frac{a_{p}}{5 \mathrm{AU}}\right)^{21 / 20} \mathrm{M}_{\oplus} / \mathrm{yr}$

Substituting eq. (3) into this equation,

$$
\dot{M}_{\text {solid }} \simeq 0.9 \times 10^{-14} f_{d}\left(\frac{\rho_{\mathrm{p}}}{1 \mathrm{gcm}^{-3}}\right)^{-1 / 6}\left(\frac{\tau_{\mathrm{damp}}}{10^{4} \mathrm{yrs}}\right)^{7 / 10}\left(\frac{M}{M_{\oplus}}\right)^{16 / 3}\left(\frac{a_{p}}{5 \mathrm{AU}}\right)^{21 / 20} \mathrm{M}_{\oplus} / \mathrm{yr}
$$

For $\tau_{\text {damp }}=10^{6} \mathrm{yrs}, f_{d} \simeq 2.5, a_{p}=5.2 \mathrm{AU}$ and $M \simeq 10 M_{\oplus}, \dot{M}_{c} \simeq 1.1 \times 10^{-7} M_{\oplus} / \mathrm{yr}$, which is one order smaller than the planetesimal accretion rate that Pollack et al. (1996) assumed.

We examine the possibility of phase 2 for other $f_{d}$ and $a_{p}$. For phase 2 to occur, $\dot{M}_{\mathrm{c}}$ must be maintained to be as large as $\dot{M}$ for $M_{\mathrm{c}} \sim M_{\text {c,hydro. Core mass can be approximately }}$ identified by core isolation mass beyond the ice line (Kokubo \& Ida 1998, 2002; Ida \& Lin 2004),

$$
M_{\mathrm{c}, \text { iso }} \simeq 4.6 f_{d}^{3 / 2}\left(\frac{a_{p}}{5 \mathrm{AU}}\right)^{3 / 4} M_{\oplus}
$$


From eq. (1) with the exponent derived by assuming eq. (33), the accretion rate required by occurrence of phase 2 is

$$
\dot{M}_{\text {solid }, 2} \simeq 10^{-6}\left(\frac{M_{\mathrm{c}}}{10 M_{\oplus}}\right)^{4.5} M_{\oplus} / \mathrm{yr} \sim 3 \times 10^{-8} f_{d}^{6.75}\left(\frac{a_{p}}{5 \mathrm{AU}}\right)^{3.4} M_{\oplus} / \mathrm{yr} .
$$

Substituting $M_{\mathrm{c}, \text { iso }}$ into $M$ in eq. (27),

$$
\eta \simeq 1.3 \times 10^{-4} f_{d}^{5}\left(\frac{\tau_{\mathrm{damp}}}{10^{4} \mathrm{yrs}}\right)^{1 / 2}\left(\frac{a_{p}}{5 \mathrm{AU}}\right)^{13 / 4}
$$

So, $\eta<1$ is equivalent to

$$
f_{d}<3.8\left(\frac{\tau_{\text {damp }}}{10^{6} \mathrm{yrs}}\right)^{-1 / 10}\left(\frac{a_{p}}{5 \mathrm{AU}}\right)^{-13 / 20}
$$

For this range of $f_{d}$ and $a_{p}>3 \mathrm{AU}$ (the ice line), eq. (28) with $M$ replaced by $M_{\mathrm{c}, \text { iso }}$ is always smaller than $\dot{M}_{\text {solid,2 }}$ given by eq. (31) (see figure 9). For $\eta>1$, on the other hand,

$$
\dot{M}_{\text {solid }} \simeq 1.5 \times 10^{-5} f_{d}\left(\frac{\rho_{\mathrm{p}}}{1 \mathrm{gcm}^{-3}}\right)^{-1 / 6}\left(\frac{\tau_{\mathrm{g}, \mathrm{acc}}}{10^{4} \mathrm{yrs}}\right)^{-4 / 5}\left(\frac{M}{M_{\oplus}}\right)^{2 / 5}\left(\frac{a_{p}}{5 \mathrm{AU}}\right)^{6 / 5} \mathrm{M}_{\oplus} / \mathrm{yr}
$$

In the range of $f_{d}$ and $a_{p}$ that satisfy $\eta>1$, eq. (34) can reach $\dot{M}_{\text {solid,2 }}$ only at $a_{p}>15 \mathrm{AU}$ and $f_{d} \sim 1$, in which gas giant formation is unlikely (Ida \& Lin 2004). Thus, the predicted $\dot{M}_{c}$ never reaches the values required for phase 2 . We conclude that phase 2 is not likely to occur for formation of giant planets. This conclusion is consistent with the ubiquity of extrasolar gas giant planets.

\section{Conclusion}

We have investigated the planetesimal accretion rate onto growing giant planets through numerical simulations and analytical arguments. The planet mass $(M)$ is increased with assumed gas accretion rate onto the planet, and orbits of planetesimals in the vicinity of the planet's orbit are integrated with the effect of gas drag, but without self-gravity of the planetesimals.

We first performed simulations with several different artificial gas accretion rates to clarify intrinsic physics determining the planetesimal accretion rate. A gap in the planetesimal disk is opened by a coupling effect of gravitational scattering by the planet and gas drag damping. Here, the gap formation means that most planetesimals are get out of the feeding zone of the planet. The scattering increases both $e$ and $b$ keeping Jacobi energy constant, 
where $e$ is orbital eccentricity and $b$ is difference in semimajor axis between the planet and the planetesimals. Changes in $e$ and $b h$ are of the same order, where $h$ is reduced Hill radius defined by $\left(M / 3 M_{*}\right)^{1 / 3}$. Since the gas drag predominantly damps $e$ after the scattering, the gap is formed. On the other hand, the width of the feeding zone is proportional to $h$. Thus, the planet growth inhibits gap formation and competes with the scattering/damping process.

We derived the condition for the gap formation by comparison between the eccentricity damping rate $\left(v_{\text {damp }}\right)$ and the rate of expansion of the feeding zone due to the planet growth $\left(v_{\mathrm{H}}\right)$. When $v_{\mathrm{H}} / v_{\text {damp }}>1$, the gap is not formed. Then, the planetesimal accretion rate $\left(d M_{\text {solid }} / d t\right)$ is scaled by the ratio of the scattering rate $v_{\text {scat }}$ to $v_{\mathrm{H}}$. The numerical results are fitted as

$$
\frac{d M_{\text {solid }}}{d t}=10^{-6}\left(\frac{R}{R_{\oplus}}\right)^{2} f_{d}\left(\frac{v_{\mathrm{H}}}{v_{\text {scat }}}\right)^{0.8} \mathrm{M}_{\oplus} \mathrm{yr}^{-1},
$$

where $R$ is physical radius of the planet and $f_{d}$ is a scaling factor for surface density of the planetesimals (eq. [12]). When the gap is formed $\left(v_{\mathrm{H}} / v_{\text {damp }}<1\right)$, the accretion rate is significantly depleted. We found that the accretion rate is scaled by $v_{\mathrm{H}} / v_{\text {damp }}$ as

$$
\frac{d M_{\text {solid }}}{d t}=10^{-6}\left(\frac{R}{R_{\oplus}}\right)^{2} f_{d}\left(\frac{v_{\mathrm{H}}}{v_{\text {damp }}}\right)^{1.4} \mathrm{M}_{\oplus} \mathrm{yr}^{-1}
$$

Applying these formulae to the more realistic gas accretion models described in $\S 2$, we found the followings:

1. In early stages when $M \sim O(10) M_{\oplus}$, a gap is opened in the planetesimal disk. The planetesimal accretion rate is smaller than that required for phase 2 to occur. This ensures efficient formation of gas giants, which may be consistent with the ubiquity of extrasolar giant planets.

2. In later stages $\left(M \gtrsim O(100) M_{\oplus}\right)$, the expansion of the feeding zone overwhelms the gap opening process, so the gap is filled. Then, the planetesimal accretion becomes efficient.

3. The amount of infalling planetesimals into the envelopes of Jupiter and Saturn in the late stages can be as large as several $M_{\oplus}$, which may be consistent with interior models for these planets.

In this "realistic" model, we assumed that planetesimals are infinitely supplied. However, if the accreted mass is significant, planetesimals distributed in the regions inside isolated strong mean motion resonances can be consumed. In that case, release of planetesimals 
from the resonance capture by resonance overlapping due to planet mass increase may also become a important factor (Zhou \& Lin 2007).

Guillot et al. (2006) pointed out the correlation that the amount of solid components of extrasolar transiting gas giants increases with metallicity of their host stars that is proportional to $f_{d}$. This trend is consistent with our formulae, because $d M_{\text {solid }} / d t \propto f_{d}$. As this example shows, the analysis here will give deep insights into formation of extrasolar gas giants and their diversity.

This work is supported by JSPS.

\section{Appendix}

The magnitude of $\left(e / h^{2}\right)$ in $\S 4.3$ is determined by a balance between damping due to the gas drag and excitation due to the planet's perturbations. Since in the non-gap case, planetesimals are engulfed by the feeding zone mainly through the parameter range of $(e / h) \lesssim 1$, we use eq. (21) for definition of the parameter $\xi$. However, gap opening is caused by damping of relatively high orbital eccentricity, so we use the formula of excitation of planetesimal eccentricity due to the protoplanet's perturbations for $(e / h) \gtrsim 1$, in evaluating $(e / h)^{2}$. Then the scattering timescale is given approximately by Chandrasekahr's two-body scattering formula (e.g., Stewart \& Ida 2000; Ohtsuki et al. 2002),

$$
\tau_{e, \mathrm{scat}} \simeq \frac{1}{n_{p} \pi\left(G M /\left(e v_{\mathrm{K}}\right)^{2}\right)^{2} e v_{\mathrm{K}} \ln \Lambda},
$$

where $\ln \Lambda \sim 3$ and $n_{p}$ is spatial density of the protoplanet, which is given by inverse of volume of the planetesimal disk in the feeding zone, $1 /\left(2 \pi a_{p} \times 4 \sqrt{3} h a_{p}\right)\left(e v_{\mathrm{K}} / \Omega_{\mathrm{K}}\right)$. Then,

$$
\tau_{e, \mathrm{scat}} \simeq \frac{8 \sqrt{3} \pi(e / h)^{4}}{27 \pi} h^{-1} \frac{T_{\mathrm{K}}}{2 \pi} \simeq 1 \times 10^{2}(e / h)^{4}\left(\frac{M}{M_{\oplus}}\right)^{-1 / 3}\left(\frac{a_{p}}{5 \mathrm{AU}}\right)^{3 / 2} \mathrm{yrs} .
$$

From $\tau_{e, \text { scat }}=\tau_{\text {damp }}$, we obtain

$$
(e / h)^{2} \simeq 10\left(\frac{\tau_{\text {damp }}}{10^{4} \mathrm{yrs}}\right)^{1 / 2}\left(\frac{M}{M_{\oplus}}\right)^{1 / 6}\left(\frac{a_{p}}{5 \mathrm{AU}}\right)^{-3 / 4} .
$$

\section{REFERENCES}

Adachi, I., Hayashi, C., \& Nakazawa, K. 1976, Prog. Theor. Phys., 56, 1756 
Fortier, A., Benvenuto, O. G. \& Brunini, A. 2007, A\&A, 473, 311.

Guillot, T., Santos, N. C., Pont, F., Iro, N., Melo, C. \& Ribas, I. 2006, A\&A, 473, L21.

Goldreich, P., \& Tremaine, S. 1982, Ann. Rev. Astron. Astrophys. 20, 249

Hasegawa, M. \& Nakazawa, K. 1990, Astro. Astrophys, 227, 619

Hayashi, C., Nakazawa, K., \& Adachi, I. 1977, Publ. Astron. Soc. Jpn., 2, 163

Hayashi, C. 1981, Prog. Theor. Phys. Suppl., 70, 35

Ida, S. 1990, Icarus, 88, 129

Ida, S., \& Lin, D. N. C. 2004, ApJ, 604, 388

Ida, S., \& Lin, D. N. C. 2008, ApJ, 673, 487

Ikoma, M., \& Genda, H. 2006, ApJ, 650, 1150

Ikoma, M., Nakazawa, K., \& Emori, H. 2000, ApJ, 537, 1013

Kokubo, E., \& Ida, S. 1998, Icarus, 131, 171

Kokubo, E., \& Ida, S. 2002, ApJ, 581, 666

Lin, D. N. C., \& Papaloizou, J. C. B. 1993, in Protostars and Planets III, ed. E. H. Levy and J. I. Lunine (Tucson:Univ. of Arizona Press), 749

Makino, J. 1991, Publ. Astron. Soc. Jpn., 4, 859

Makino, J., \& Aarsetth, S.J. 1992, Publ. Astron. Soc. Jpn., 44, 141

Mizuno, H. 1980, Prog. Theor. Phys. Suppl., 64, 54

Nakazawa, K., Ida, S. 1988, Prog. Theor. Phys. Suppl., 96, 167

Ohtuski, K., Stewart G. R. \& Ida, S. 2002, Icarus, 155, 436

Podolak, M. 2003, Icarus, 165, 428

Pollack, J. B., McKay, C. P., \& Christofferson, B. M. 1985, Icarus, 64, 471

Pollack, J. B., Hubickyj, O., Bodenheimer, P., Lissauer, J.J., Podolak, M., \& Greenzweig, Y. 1996, Icarus, 124, 62

Saumon, D., Guillot, T. 2004, ApJ, 609, 1170 
Stevenson, D. J. 1982, P\&SS, 30, 755

Stewart, G. R. \& Ida, S. 2000, Icarus, 143, 28

Tanaka, H., \& Ida, S. 1997, Icarus, 125, 302

Tanaka, H., \& Ida, S. 1999, Icarus, 139, 350

Tanaka, H. \& Ward, W. R., 2004, ApJ, 602, 388

Tanigawa, T., \& Watanabe, S. 2002, ApJ, 580, 506

Thommes, E. W., Duncan, M. J. \& Levison, H. F. 2003, Icarus, 161, 431.

Zhou, J.-L, Lin, D. N. C. 2007, ApJ, 666, 447 


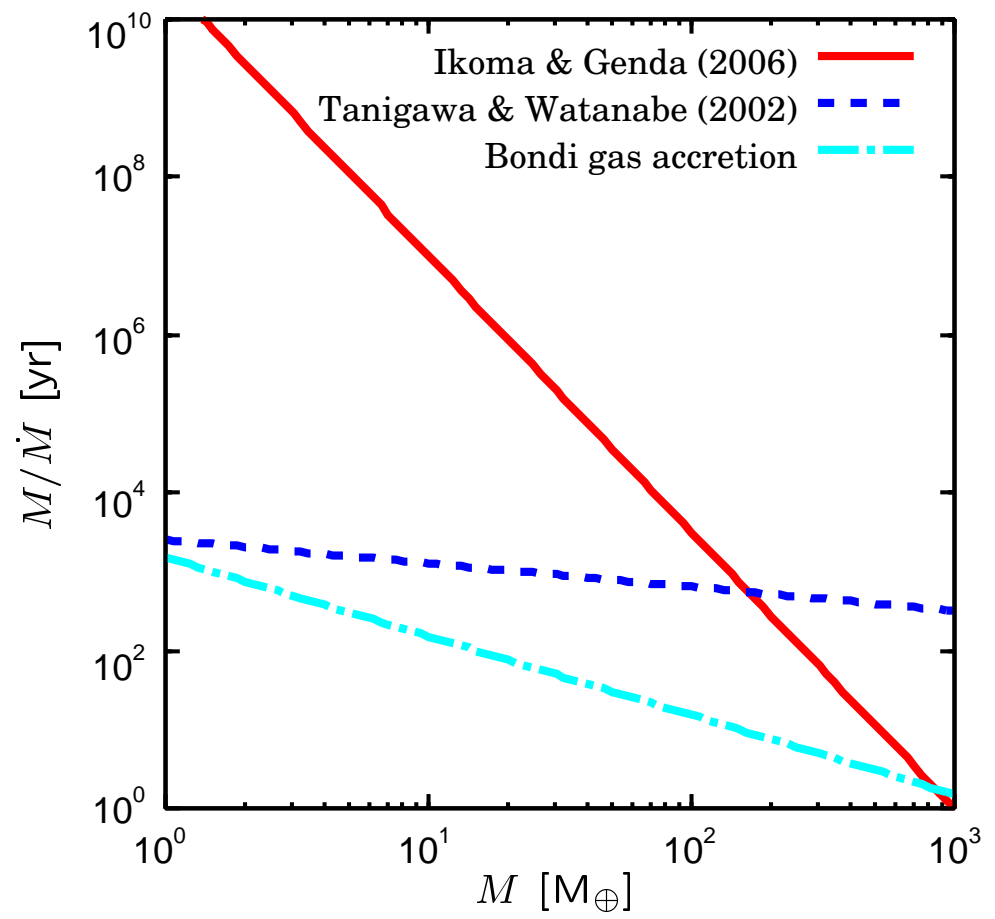

Fig. 1. - Gas accretion timescales for a planet with mass $M$ at $a=5 \mathrm{AU}$. Solid line is an extrapolation of the model by Ikoma \& Genda (2006). Dashed and dotted-dashed lines represent limits by Tanigawa \& Watanabe (2002) and Bondi accretion. 


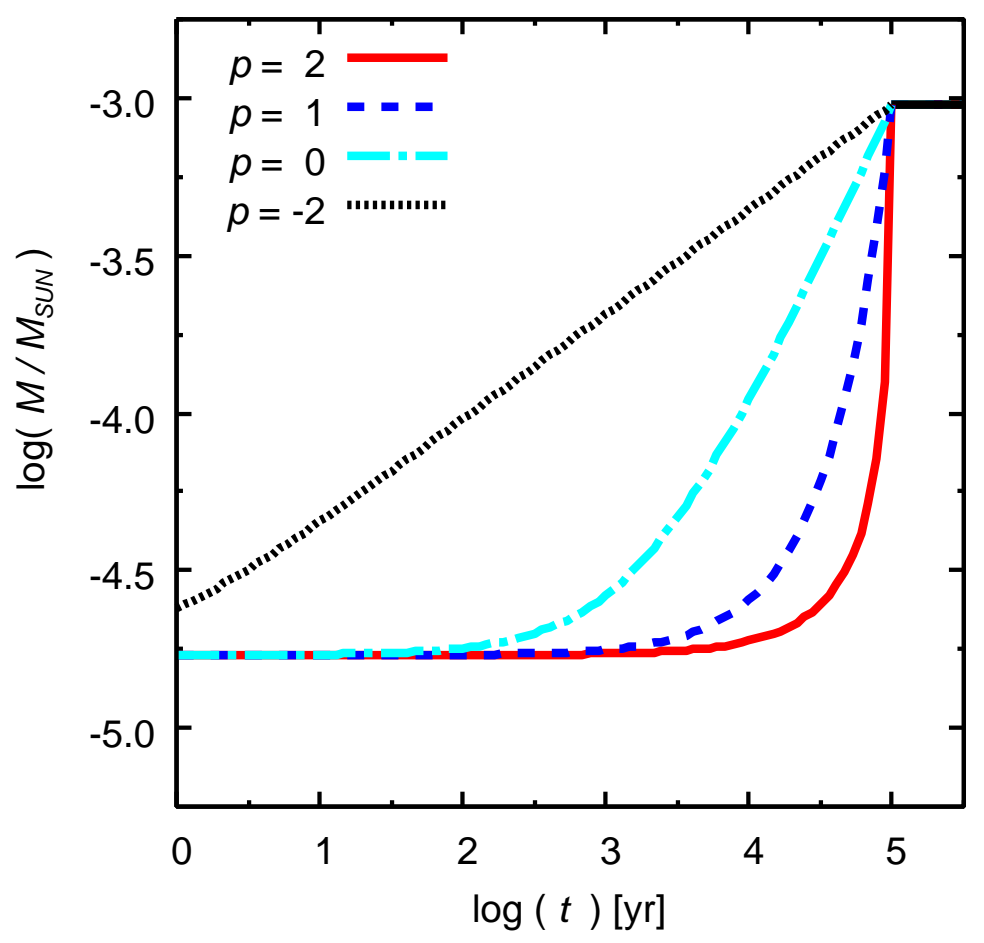

Fig. 2.- Evolution of the protoplanet mass according to the simple power-law gas accretion models $\left(\dot{M} \propto M^{p}\right)$. Initial and final masses are $M_{0}=5.67 M_{\oplus}$ and $M_{\mathrm{f}}=M_{J}$, where $M_{J}\left(=10^{-3} M_{\odot}\right)$ is a Jupiter mass. Growth timescale $t_{\mathrm{f}}=10^{5} \mathrm{yr}$. After $t>t_{\mathrm{f}}$, we set $M=M_{\mathrm{f}}=$ const. 

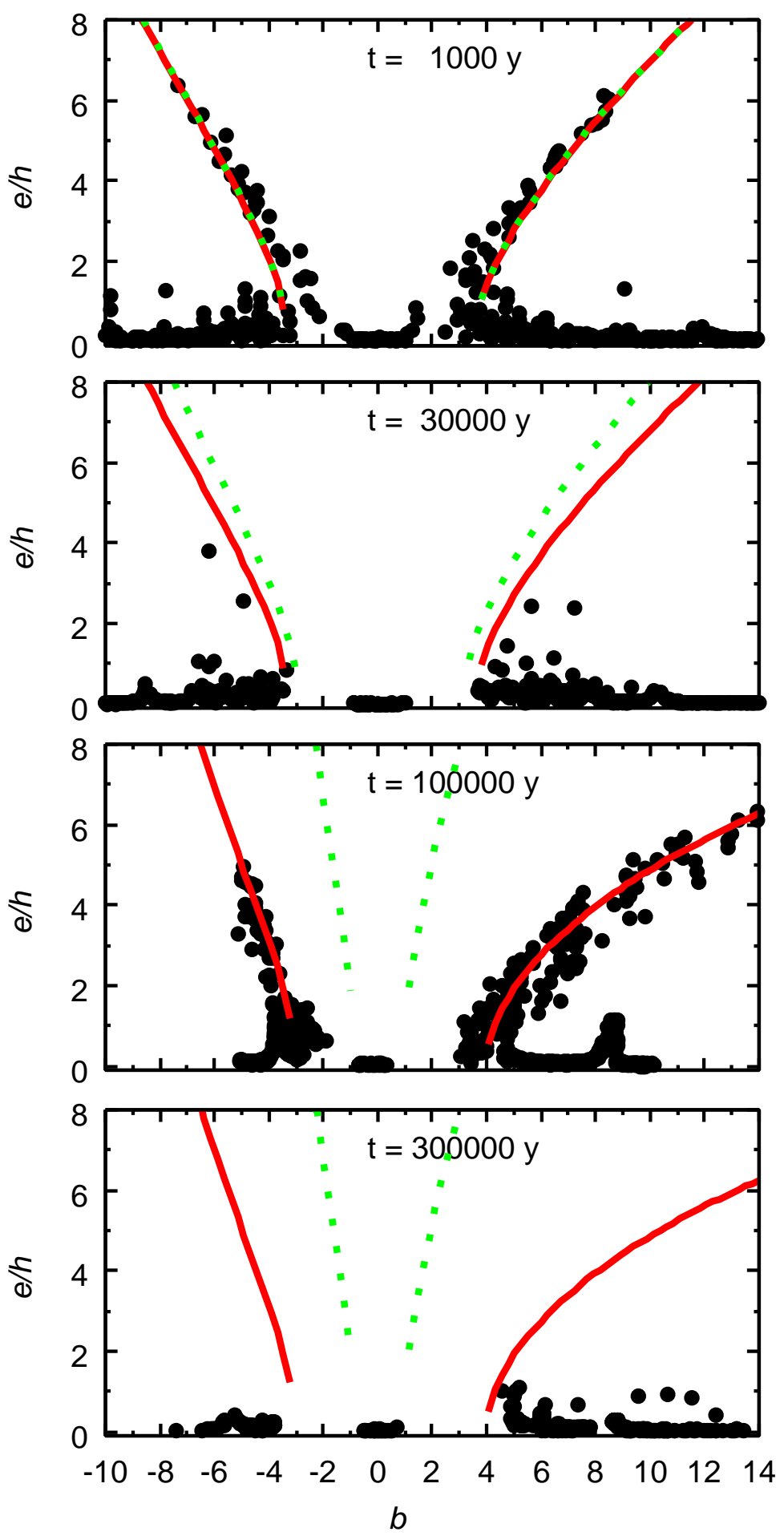

Fig. 3.- Orbital evolution of a swarm of a planetesimals on the $b-(e / h)$ plane. We adopt $p=2, \tau_{\text {damp }}=10^{4} \mathrm{yrs}$ and $t_{\mathrm{f}}=10^{5} \mathrm{yrs}$. The planet is fixed at $e / h=b=0\left(a_{p}=5 \mathrm{AU}\right)$. The horizontal axis $b$ expresses $\left(a-a_{p}\right) / h$ where $a$ is the semimajor axis of planetesimals. Solid and dotted lines represent the boundaries of the feeding zone (i.e. Jacobi energy $E_{J}=0$ ) and those at $t=0$, respectively. The time evolution of the latter is caused by increase in $h$. The selected number of planetesimals is 1000 in $3.3 \mathrm{AU} \leq a \leq 8.4 \mathrm{AU}$ at $t=0$. The numbers of planetesimals are $998\left(10^{3} \mathrm{yr}\right), 992\left(3 \times 10^{4} \mathrm{yr}\right), 904\left(10^{5} \mathrm{yr}\right)$, and $878\left(3 \times 10^{5} \mathrm{yr}\right)$. 

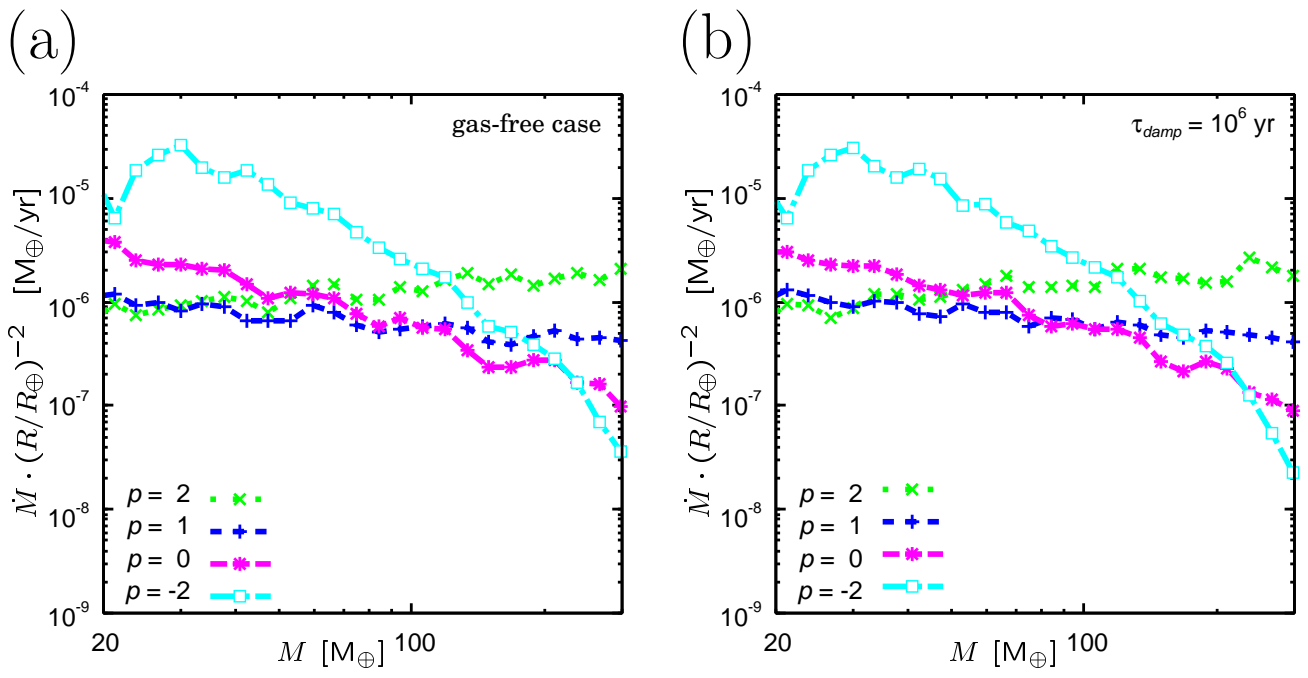

(c)

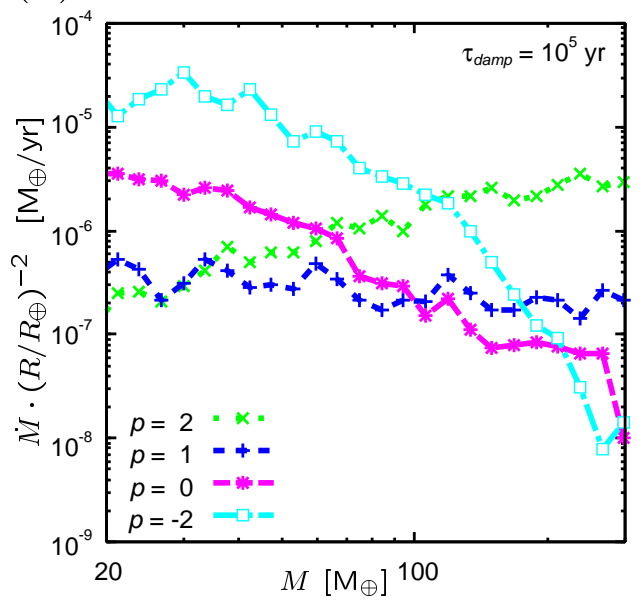

(d)

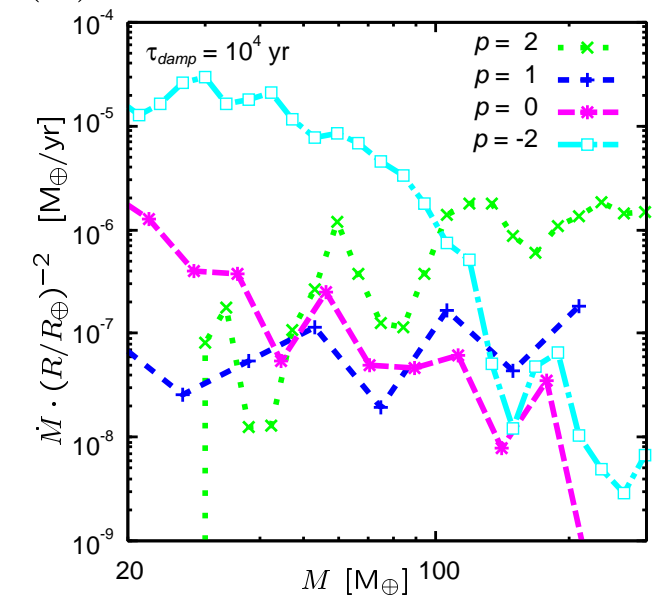

Fig. 4.- Evolution of the planetesimal accretion rate onto the growing planet as a function of the protoplanet mass $(M)$. (a) The results in the gas-free case, the cases of (b) $\tau_{\text {damp }}=10^{6}$ yrs, (c) $10^{5}$ yrs and (d) $10^{4}$ yrs. The four lines in the each panel represent the results with various gas accretion models $(p=2,1,0,-2)$. Initial mass of the protoplanet $M_{0}$ is set as $5.67 M_{\oplus}$. The systems initially consist of 20,000 planetesimals, so the individual planetesimal masses correspond to $\simeq 7.7 \times 10^{-4} M_{\oplus}$. 
(a)

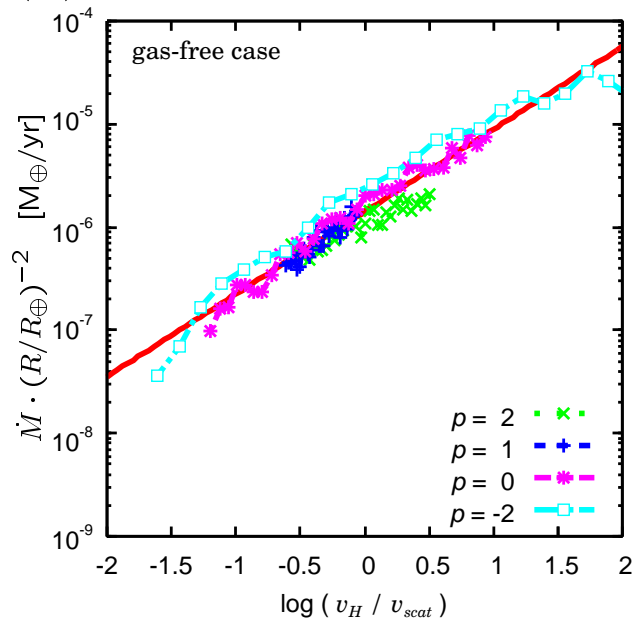

(c)

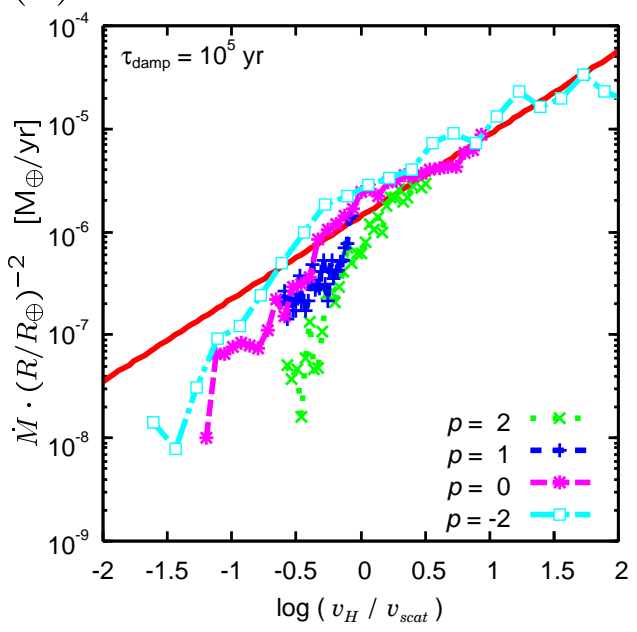

(b)

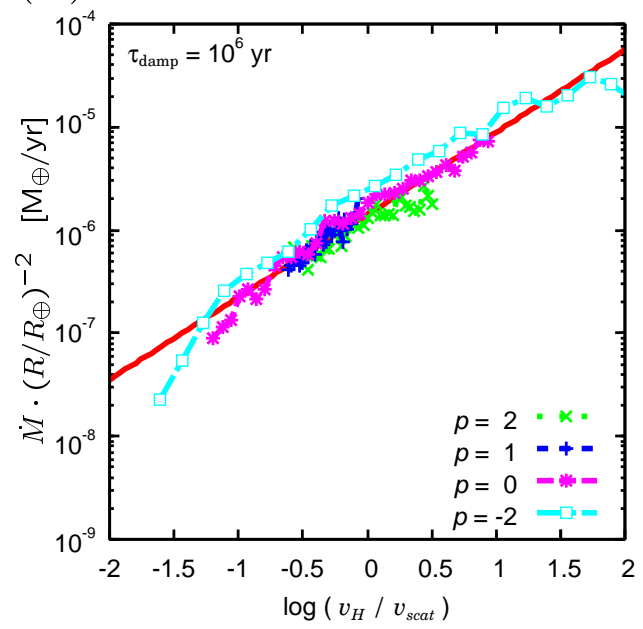

(d)

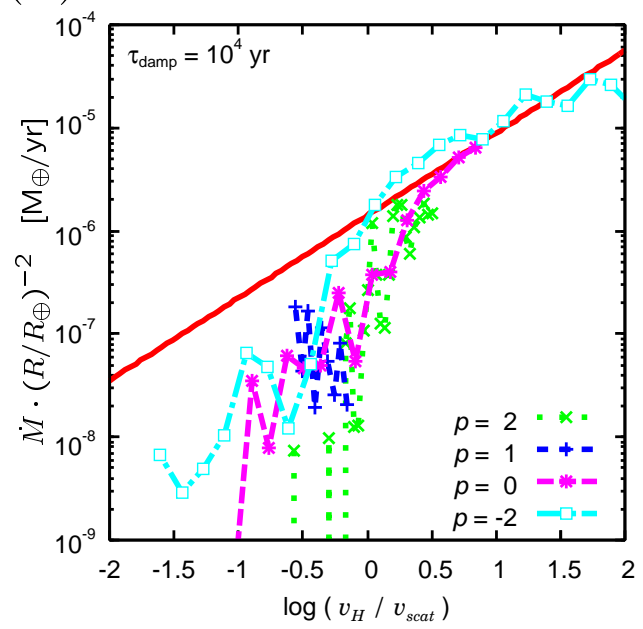

Fig. 5.- Evolution of the planetesimal accretion rate as a function of $\xi=v_{H} / v_{\text {scat }}$ for $p=2,1,0$ and -2 . (a) The results in the gas-free case, the cases of (b) $\tau_{\text {damp }}=10^{6} \mathrm{yrs}$, (c) $10^{5} \mathrm{yrs}$ and (d) $10^{4} \mathrm{yrs}$. The fitting formula, eq. (24), is expressed by thick solid lines in the plots. 

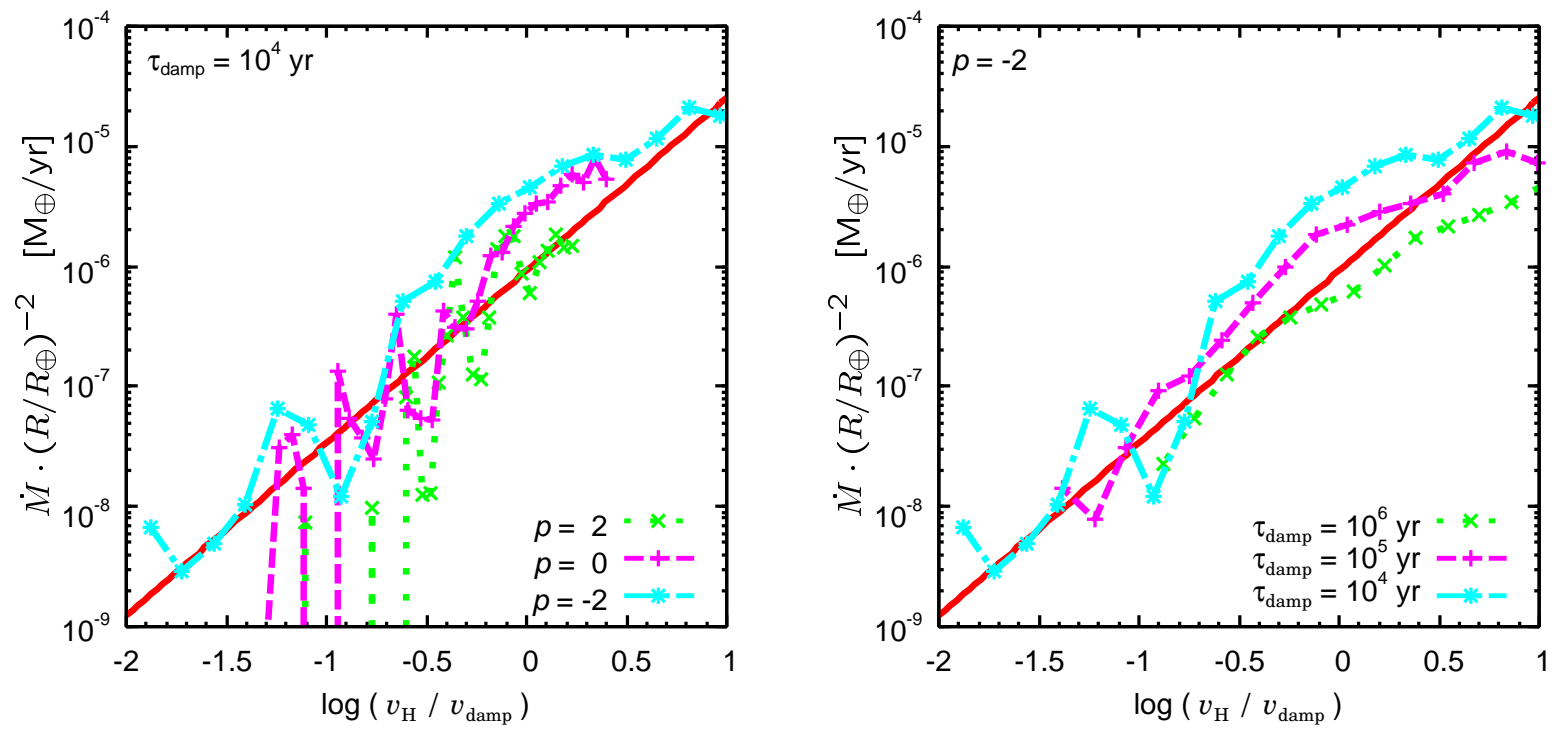

Fig. 6. - Evolution of the scaled planetesimal accretion rate as a function of $\eta=v_{H} / v_{\text {damp }}$ for $p=2,0$ and -2 in the case of $\tau_{\text {damp }}=10^{4} \mathrm{yrs}$ (left panel) and for $\tau_{\text {damp }}=10^{6}, 10^{5}$ and $10^{4}$ yrs in the case of $p=-2$ (right panel). The fitting formula, eq. (25), is expressed by thick solid lines. 

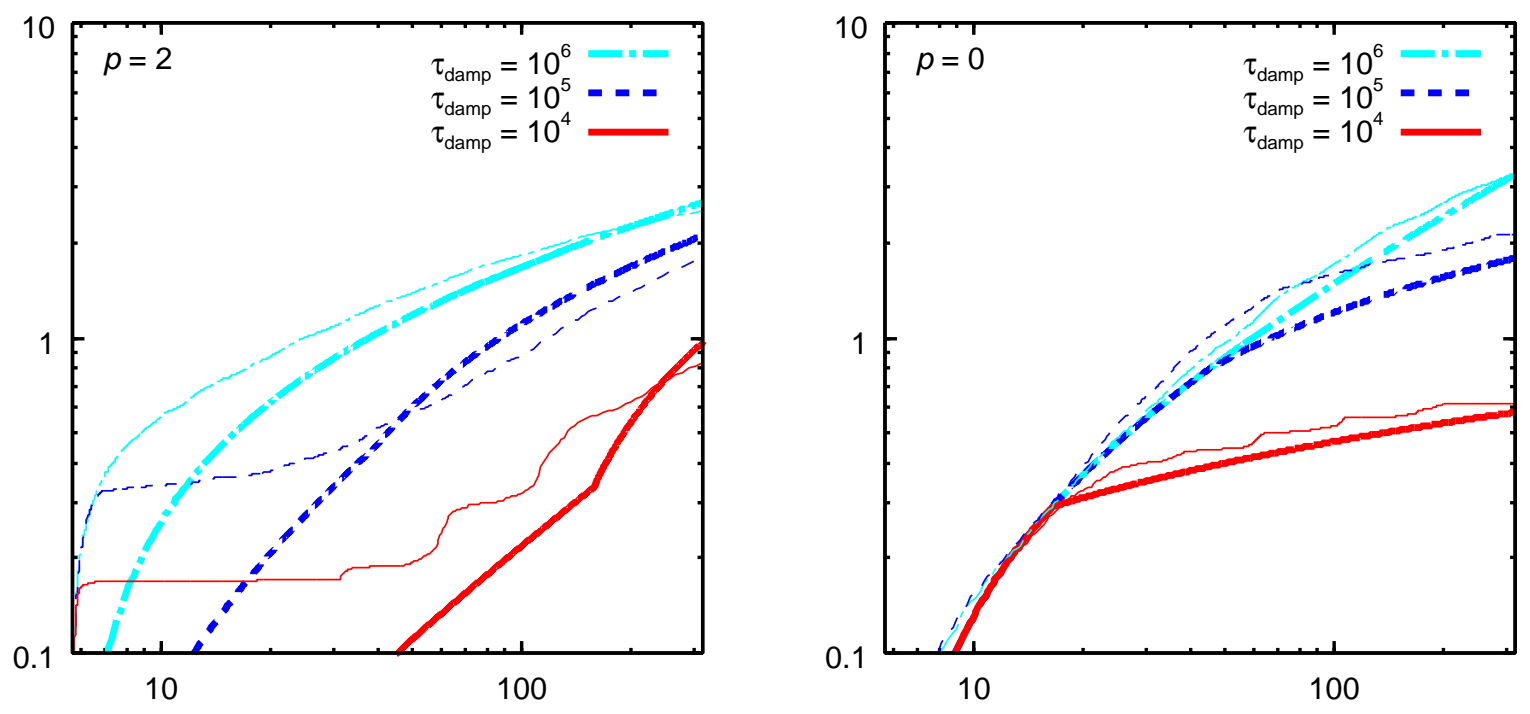

Fig. 7.- Comparison between the numerical simulations and the semi-analytical results. The left and right panels show the results for gas accretion with $p=2$ and $p=0$, respectively. The thin and thick curves represent the numerical and semi-analytical results. 

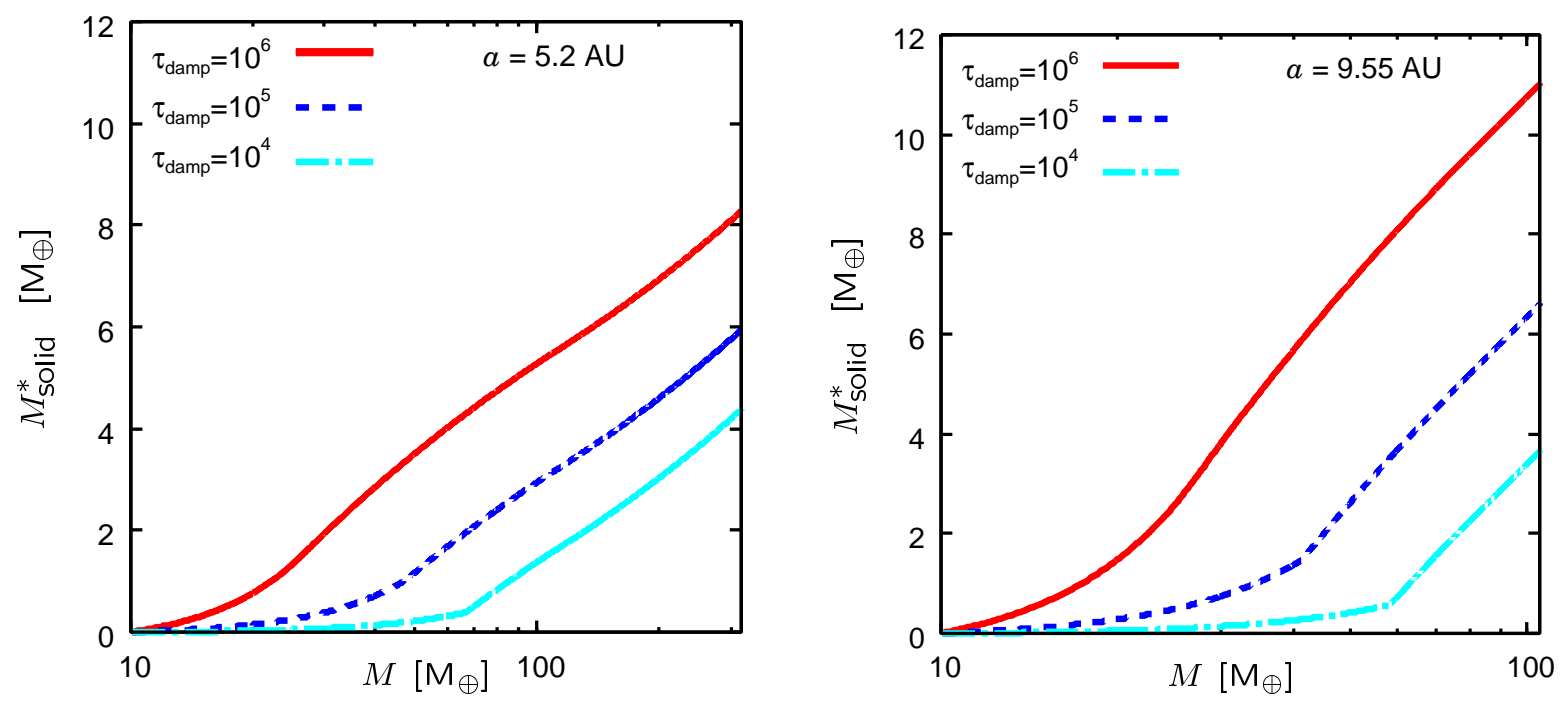

Fig. 8.- The evolution of cumulative mass of accreted planetesimals as a function of $M$ in the case with $M_{0}=10 M_{\oplus}$. The left and right panels show the results for $a=5.2 \mathrm{AU}$ and $a=9.55 \mathrm{AU}$, which correspond to Jupiter and Saturn. Here, $R=2 R_{1}$ and $f_{d}=2$ are assumed, where $R_{1}$ is the physical radius for mass $M$ and $\rho=1 \mathrm{gcm}^{-3}$. For other $R$ and $f_{d}$, the accreted mass is multiplied by $\left(R / 2 R_{1}\right)^{1 / 2}\left(f_{d} / 2\right)$. 


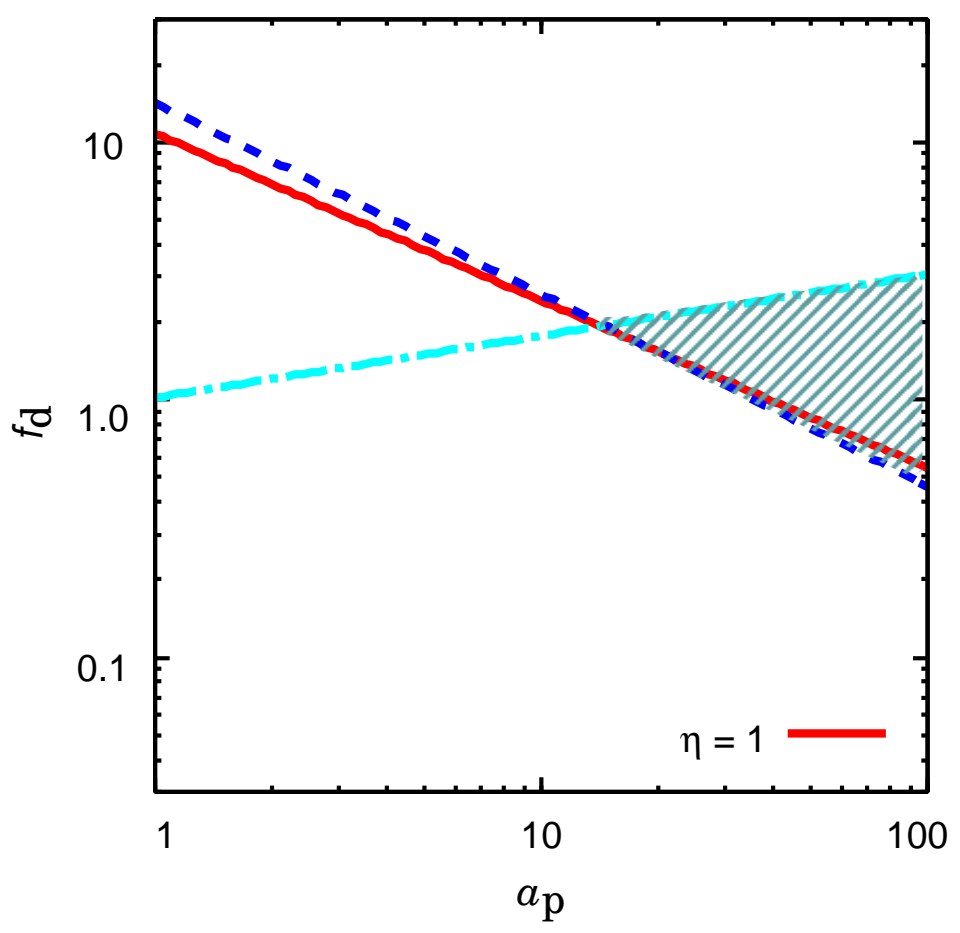

Fig. 9.- The parameter range in which phase 2 can occur, which is expressed by the shaded regions. The region above the solid line represents $\eta>1$. Phase 2 can occur in the regions above the dashed line for $\eta<1$ and in the regions below the dot-dashed line for $\eta>1$. 